\title{
Differential DNA methylation of the D4Z4 repeat in patients with FSHD and asymptomatic carriers
}

Marie-Cécile Gaillard, MS

Stéphane Roche, PhD

Camille Dion, MS

Armand Tasmadjian, BS

Gwenaëlle Bouget, MS

Emmanuelle Salort-

Campana, MD

Catherine Vovan, BS

Charlene Chaix, BS

Natacha Broucqsault, $\mathrm{PhD}$

Julia Morere, $\mathrm{PhD}$

Francesca Puppo, PhD

Marc Bartoli, PhD

Nicolas Levy, MD, PhD

Rafaëlle Bernard, MD

Shahram Attarian, MD, $\mathrm{PhD}$

Karine Nguyen, MD, $\mathrm{PhD}$

Frédérique Magdinier, $\mathrm{PhD}$

\section{Correspondence to}

Dr. Magdinier:

frederique.magdinier@univ-amu.fr

\section{ABSTRACT}

Objective: We investigated the link between DNA hypomethylation and clinical penetrance in facioscapulohumeral dystrophy (FSHD) because hypomethylation is moderate and heterogeneous in patients and could not thus far be correlated with disease presence or severity.

Methods: To investigate the link between clinical signs of FSHD and DNA methylation, we explored 95 cases (37 FSHD1, 29 asymptomatic individuals carrying a shortened D4Z4 array, 9 patients with FSHD2, and 20 controls) by implementing 2 approaches: methylated DNA immunoprecipitation and sodium bisulfite sequencing.

Results: Both methods revealed statistically significant differences between asymptomatic carriers or controls and individuals with clinical FSHD, especially in the proximal region of the repeat. Absence of clinical expression in asymptomatic carriers is associated with a level of methylation similar to controls.

Conclusions: We provide a proof of concept that the targeted approaches that we describe could be applied systematically to patient samples in routine diagnosis and suggest that local hypomethylation within D4Z4 might serve as a modifier for clinical expression of FSHD phenotype.

Classification of evidence: This study provides Class III evidence that assays for hypomethylation within the D4Z4 region accurately distinguishes patients with FSHD from individuals with D4Z4 contraction without FSHD. Neurology ${ }^{\circledR}$ 2014;83:1-10

\section{GLOSSARY}

FSHD $=$ facioscapulohumeral dystrophy; MeDIP $=$ methylated DNA immunoprecipitation; PCA $=$ principal compound analysis.

DNA methylation, a covalent addition of methyl groups to the carbon 5 position of cytosine within CpG dinucleotides, has important roles in several physiologic processes ${ }^{1-4}$ but also contributes to a number of diseases. ${ }^{5,6}$ A large fraction of the human genome corresponds to $\mathrm{CpG}$-rich repetitive sequences, the majority of which are packed as condensed and repressed chromatin by DNA methylation. ${ }^{7}$ Hypomethylation of repetitive DNA has multiple consequences; aberrant DNA methylation of repetitive sequences has a causal role in a variety of diseases including rare constitutive diseases such as facioscapulohumeral dystrophy (FSHD) ${ }^{8,9}$

FSHD is an autosomal dominant myopathy characterized by a progressive and asymmetric weakening of facial, shoulder, and upper body muscles with a progression to the lower body. Specific groups of muscles are affected but the underlying pathomechanism is not fully understood. At the molecular level, the pathology is linked to the deletion of an integral number of repetitive macrosatellite elements ( $D 4 Z 4$; figure $1 \mathrm{~A})$ at the $4 \mathrm{q} 35$ subtelomeric locus. In the general population, the number of $D 4 Z 4$ repeats ranges from 11 to up to $100-150$ copies, whereas in most patients, this number is below the threshold of 11 copies.

D4Z4 is extremely GC rich (70\%) and methylated in normal individuals but hypomethylated in patients with FSHD $^{9-13}$ and in patients with phenotypic FSHD (FSHD2) carrying long

From Aix Marseille Universite (M.-C.G., S.R., C.D., A.T., G.B., E.S.-C., N.B., J.M., F.P., M.B., N.L., R.B., S.A., K.N., F.M.), INSERM UMR S_910, Marseille; and APHM, Centre de Référence des Maladies Neuromusculaires et de la SLA (E.S.-C., S.A.), and APHM, Laboratoire de Génétique Médicale (C.V., C.C., N.L., R.B., K.N.), Hôpital de la Timone, Marseille, France.

Go to Neurology.org for full disclosures. Funding information and disclosures deemed relevant by the authors, if any, are provided at the end of the article. 
Figure 1 Experimental procedure

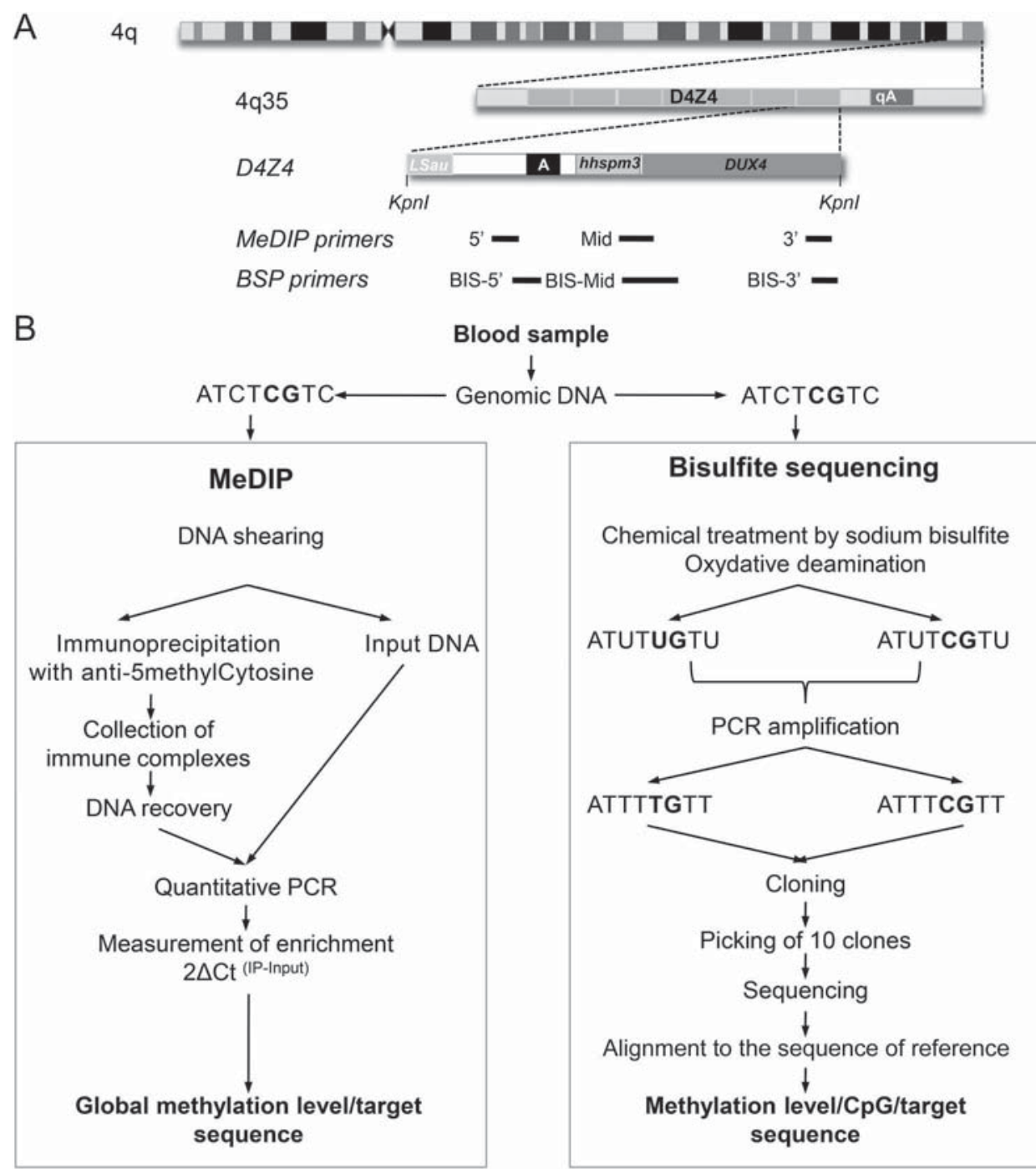

C

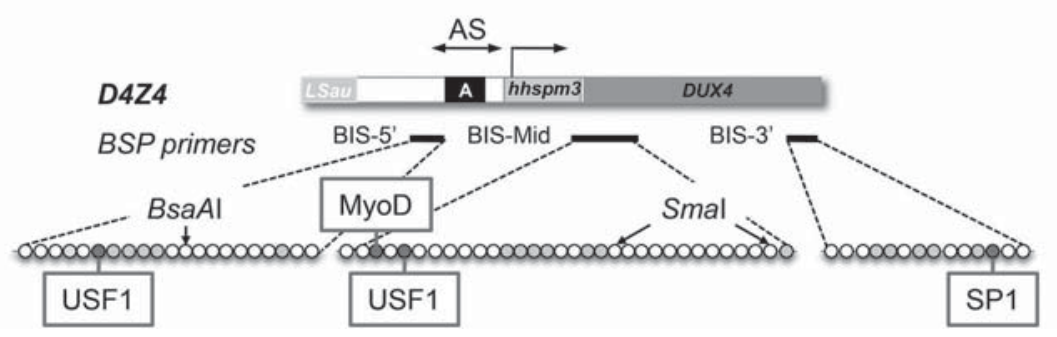

(A) Schematic representation of chromosome 4 and $4 \mathrm{q} 35$ locus. The $3.3 \mathrm{~kb} \mathrm{D4Z4} \mathrm{repeat} \mathrm{is} \mathrm{flanked} \mathrm{by} 2 \mathrm{Kpnl}$ sites. D4Z4 is extremely GC rich (70\%) and contains part of the LSau repetitive sequence, several microsatellites, hhspm3, a low GC-rich repeat, and an open reading frame encoding a double homeodomain protein. The DUX4 double homeobox sequence is represented together with other repetitive elements within D4Z4 (LSau, region A and hhspm3). The position of the primers used for methylated DNA immunoprecipitation (MeDIP) or sodium bisulfite sequencing is shown. (B) Diagrams showing the procedure used for measuring the global methylation level of D4Z4 on DNA from peripheral blood lymphocytes from the different groups (see tables e-1-e-5). DNA was either used for MeDIP with an antibody against 5-methylcytosine $(5 \mathrm{mC})$ or converted by oxidative deamination in the presence of sodium bisulfite. (C) Details of the 3 regions analyzed in this study at position 1003-1277 (BIS-5') containing the transcription start site for sense and antisense RNA (arrows); 1616-1969 (BIS-mid), encompassing the DUX4 promoter (shown by arrow), and position 3058-3228 (BIS-3') with respect to the first $\mathrm{Kpnl}$ site. The position of the methylation-sensitive restriction sites used for Southern blot is indicated. The putative binding sites for transcription factor or regulatory factors are indicated for each fragment (dark gray dots). Light gray dots correspond to other putative binding sites for factors not expressed in muscle. 
D4Z4 arrays on both chromosomes $4 .{ }^{14}$ However, the link between $D 4 Z 4$ hypomethylation and FSHD is still unclear.

METHODS To investigate the link between presence or total absence of clinical signs of FSHD and D4Z4 methylation level, we explored a cohort of 37 FSHD1, 9 FSHD2, 20 nonsymptomatic noncarrier individuals (controls), and 29 nonsymptomatic carriers of a shortened $D 4 Z 4$ array ( $<11$ units) by implementing 2 complementary approaches: methylated DNA immunoprecipitation (MeDIP) and sodium bisulfite sequencing (figure 1B). We provide Class III evidence that assays for $D 4 Z 4$ methylation distinguishes patients with FSHD from asymptomatic carriers.

Standard protocol approvals, registrations, and patient consents. All individuals provided written informed consent for the use of their DNA sample for medical research. The study was performed in accordance with the Declaration of Helsinki. Controls (table e-1 on the Neurology ${ }^{\circledR}$ Web site at Neurology.org) were either randomly selected individuals or were patients' relatives who did not in either case carry a short $D 4 Z 4$ array nor were affected by any muscular pathology (details in tables e-2-e-5 and supplementary materials). Controls were selected in the same age range and sex representation as test subjects.

Detailed e-Methods are given in the supplementary information section.

RESULTS FSHD is characterized by a high interindividual and intrafamilial variability in the age at onset and course of the disease. Penetrance of the symptoms is estimated between $80 \%$ and $95 \%$ at the age of 20 to 30 years, but significantly higher in men than women. Furthermore, an inverse correlation between the number of residual repeats and the penetrance has been proposed but numerous cases of asymptomatic individuals carrying a low number of repeats have been reported. ${ }^{15-19}$ To evaluate to what extent $D 4 Z 4$ methylation changes are associated with the presence or absence of clinical signs of FSHD, we developed and tested 2 approaches, MeDIP and sodium bisulfite sequencing, on a large cohort of clinically affected patients with FSHD carrying a shortened D4Z4 array (FSHD1), patients with phenotypic FSHD (FSHD2), and controls. Furthermore, we also addressed whether $D 4 Z 4$ methylation might explain the discrepancy in the clinical expression of the pathology by analyzing the $D 4 Z 4$ methylation pattern in 29 asymptomatic carriers.

Among a number of protocols for measuring DNA methylation, bisulfite sequencing stands out for its reliability in quantifying the DNA methylation status at a single nucleotide resolution in heterogeneous samples. This methodology depends on the oxidative deamination of cytosines into uracils upon treatment by sodium bisulfite while methylated cytosines remain (figure $1 \mathrm{~B}$, table e-6). Bisulfite-treated samples are then amplified by PCR using sequencespecific primers designed to amplify methylated and unmethylated DNA with the same efficiency. ${ }^{20}$ The multiple DNA fragments are subcloned and sequenced allowing analysis of the methylation status/CpG/DNA molecule (figure 1B). Because bisulfite sequencing is a time-consuming and resource-intensive method, we also evaluated the level of DNA methylation with a less direct and less sensitive approach by using immunoprecipitation of methylated DNA fragments (MeDIP) followed by quantitative PCR (figure 1B, table e-6). Technical performance of both methods was first analyzed by comparing different clones of the HCT116 colon cancer cell line. We applied either bisulfite sequencing (figure 2, A-C) or MeDIP (figure 2D) to DNA from parental HCT116, HCT116 in which the DNA methyltransferase $3 \mathrm{~b}$ had been invalidated (HCT116 3b KO), or HCT116 in which both DNA methyltransferases 1 and $3 \mathrm{~b}$ had been invalidated (HCT116 dKO). ${ }^{21}$ We observed an almost complete hypomethylation of $D 4 Z 4$ in the $\mathrm{dKO}$ cells compared with the parental cells by both methods. We observed an intermediate methylation pattern for the $3 \mathrm{~b} \mathrm{KO}$ cells suggesting that both DNA methyltransferases are required to maintain a high level of methylation along the $D 4 Z 4$ sequence but also for 2 other macrosatellite repeats (DXZ4 on chromosome $\mathrm{X}$, RS447 on chromosome 4).

Then, to evaluate the link between DNA methylation and the presence or absence of clinical signs of FSHD, we investigated the level of DNA methylation by MeDIP followed by quantitative PCR for 3 regions within D4Z4 in 37 FSHD1 cases (number of repeats ranging from 2 to 10), 29 asymptomatic carriers in the same range for D4Z4 units, 9 phenotypic FSHD (FSHD2) cases, and 20 controls (figure 3A). Using a nonparametric pairwise comparison, we determined that enrichment in methyl groups is significantly decreased in patients with phenotypic FSHD (FSHD2) and FSHD1 compared with asymptomatic carriers. In addition, we performed a multivariate analysis (principal compound analysis [PCA]) to evaluate the correlations between the level of methylation in the different $D 4 Z 4$ subregions with sex, age, number of repeats, and presence or absence of symptoms in the 4 different subgroups of samples. As expected, the presence of symptoms correlated with a low number of D4Z4 repeats (Pearson correlation coefficient, $r=-0.333$; $p=0.004$ ). A low methylation level in the $5^{\prime}$ and $3^{\prime}$ regions of $D 4 Z 4$ was observed in patients with clinical FSHD symptoms (Pearson correlation coefficient, $r=-0.547 ; p<0.0001$, and $r=-0.446$; $p<0.0001$, respectively). By comparing the different groups, we did not observe any strong correlation $(>0.5)$ between hypomethylation and age or sex. The MeDIP data do suggest that the midregion is more methylated in females than in males $(r=-0.308$; $p=0.008)$, but that sex may also correlate with 


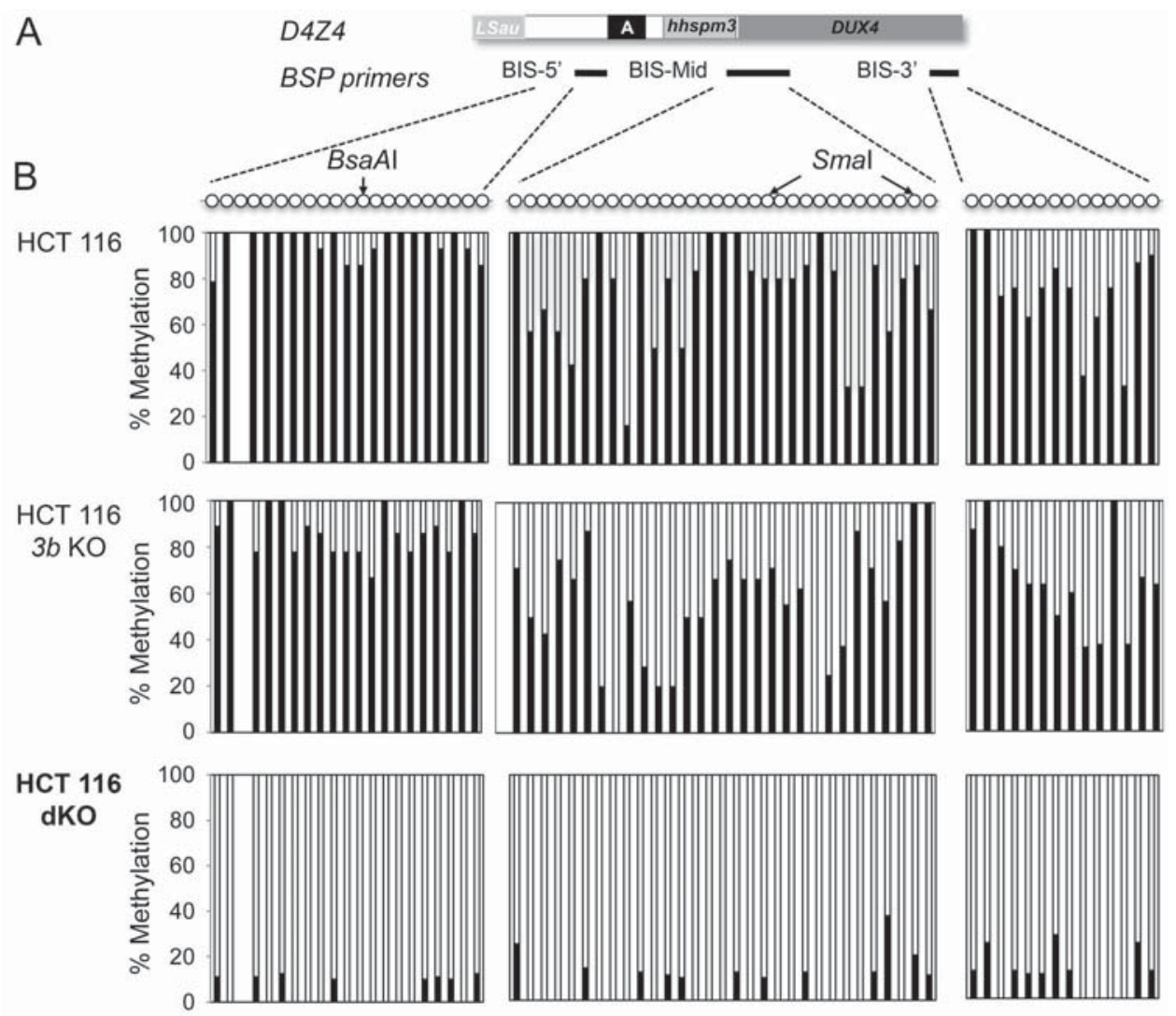

C
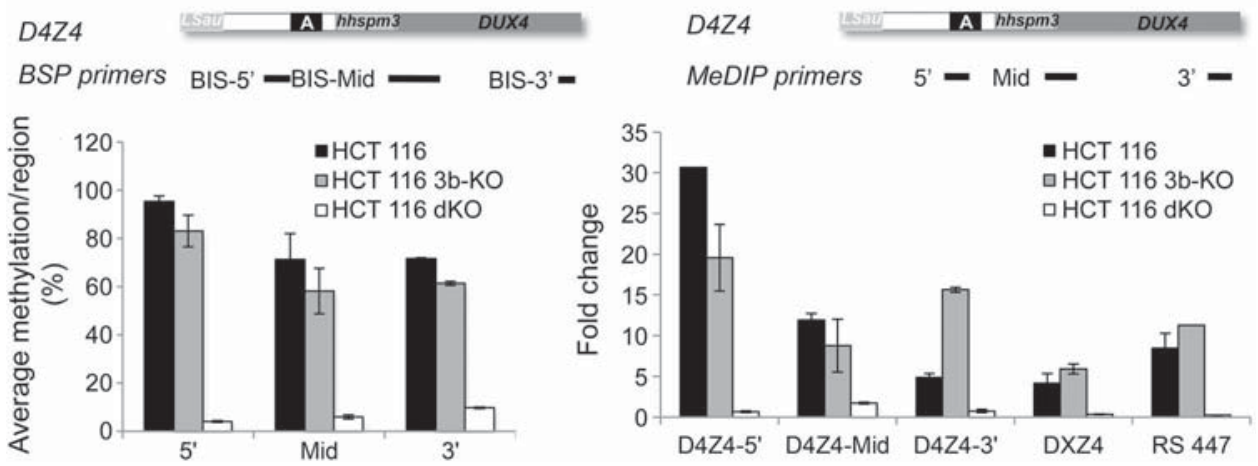

(A) Three different regions within D4Z4 were amplified by PCR after sodium bisulfite sequencing of total genomic DNA. (B) Amplicons (BIS-5', 21 CpGs, BIS-mid, 31 CpGs, BIS-3', 14 CpGs) were cloned and at least 10 individual clones were analyzed by Sanger sequencing. Each clone is representative of a molecule of DNA of the initial sample. To validate the sensitivity of the method, we used the HCT116 colon cell lines (upper panel) and 2 clones derived from this cell line, HCT116 $3 \mathrm{~b}$ KO in which DNMT3b has been invalidated (middle panel) and HCT116 dKO in which both DNMT1 and $3 b$ are invalidated (lower panel). (C) The position of the 3 sets or primers used for sodium bisulfite sequencing is indicated. Histogram bars represent the percentage of methylated (black) or unmethylated (white) CpG for each position in the 5' (21 CpGs), mid (31 CpGs), and 3' (14 CpGs) sequences determined by sodium bisulfite sequencing. (D) The position of the 3 sets or primers used for methylated DNA immunoprecipitation (MeDIP) is indicated. After immunoprecipitation, DNA was amplified by quantitative PCR and enrichment in methylation/CpG within the target sequence was calculated by using the $2 \Delta^{\mathrm{Ct}}$ method between the input (total DNA after shearing) and immunoprecipitated DNA. Values were normalized to a single copy intergenic sequence on chromosome 5 containing a single $\mathrm{CpG}$ site. To validate the sensitivity of the method, we compared methylation profiles in HCT116, HCT116 3b KO, and HCT116 dKO cell lines. Experiments were done in triplicate and the SD is shown (error bars). The level of methylation was also determined by MeDIP for 2 other macrosatellite repeats (DXZ4 and RS447). 

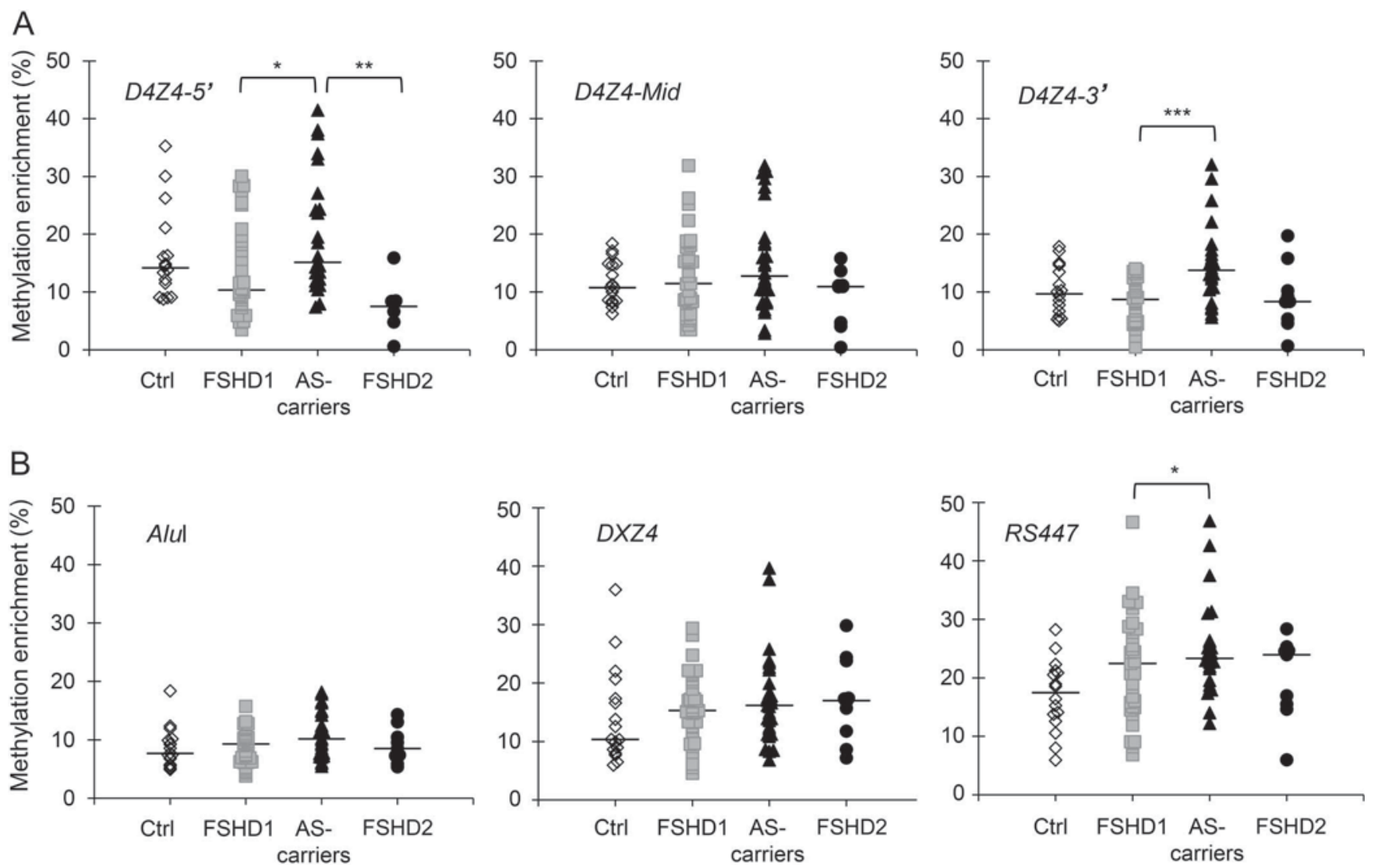

DNA methylation was determined by 2 independent methylated DNA immunoprecipitation (MeDIP) assays, and values obtained by quantitative PCR were normalized to a sequence containing a single $\mathrm{CpG}$ (chromosome 5). Four different subgroups were analyzed. Controls (Ctrl): open rhombus, $\mathrm{n}=16$; individuals with facioscapulohumeral dystrophy (FSHD)1: gray squares, $n=35$; asymptomatic carriers (AS-carriers): black triangles, $n=25$; and individuals with phenotypic FSHD (FSHD2): black circles, $n=9$. Scatterplots display the methylation enrichment/CpG in the different target sequence (y-axis). The horizontal black thick lines correspond to the median for each group. Datasets were compared with the Kruskal-Wallis nonparametric test and brackets identify groups where values are significantly different based on post hoc Dunn comparison and Bonferroni correction $(* p=0.005 ; * * p=0.001 ; * * *<$ 0.001). (A) From left to right, D4Z4 methylation level for 5', mid, and 3' in each subgroup. (B) Methylation level was determined by MeDIP for the dispersed Alul elements (left graph) and 2 other macrosatellites, DXZ4 (middle), and RS447 (right) in Ctrl, FSHD1, AS-carriers, and FSHD2.

penetrance (table e-7). Notably, this significant change in the average methylation level between symptomatic and asymptomatic FSHD is not observed genome-wide but is restricted to $D 4 Z 4$ because we did not observe any significant difference in the level of methylation for the short interspersed $A l u$ I repeats dispersed throughout the genome both in euchromatin and heterochromatin regions and reflecting the global genomic methylation (figure 3B).

Therefore, we concluded that hypomethylation is not only a function $D 4 Z 4$ residual number, but also reflects the presence of clinical signs of the disease because differences were observed between individuals with FSHD and nonaffected individuals including asymptomatic carriers and controls.

To confirm the hypothesis of a link between methylation level and clinical expression, we surveyed how methylation is transmitted within a few families comprising FSHD cases, asymptomatic carriers, and nonsymptomatic noncarriers by combining results obtained by MeDIP and/or sodium bisulfite sequencing (figure 4). Analysis of normalized MeDIP and bisulfite data confirms a decrease in methylation/ CpG at the $5^{\prime}$ end of D4Z4 in individuals affected with FSHD, which is not correlated with hypomethylation of other macrosatellites or global methylation of the genome at $A l u \mathrm{I}$ sequences.

Thus, to obtain a more precise understanding of the DNA methylation pattern in the different groups, we analyzed by sodium bisulfite sequencing 3 different regions within $D 4 Z 4$ (figure 5A) in controls, individuals with symptomatic FSHD1, asymptomatic carriers, and individuals with phenotypic FSHD. For each sample, 10 individual clones with a percentage of conversion $>95 \%$ were analyzed (figures e-1-e-3). As observed in a recent study evaluating the methylation profile of the $D 4 Z 4$ repeats in a small number of patients with phenotypic FSHD by bisulfite sequencing (i.e., 12 patients with FSHD2 carrying normalsized $4 \mathrm{qA}$ alleles), ${ }^{22}$ we observed a marked decrease in 
MeDIP

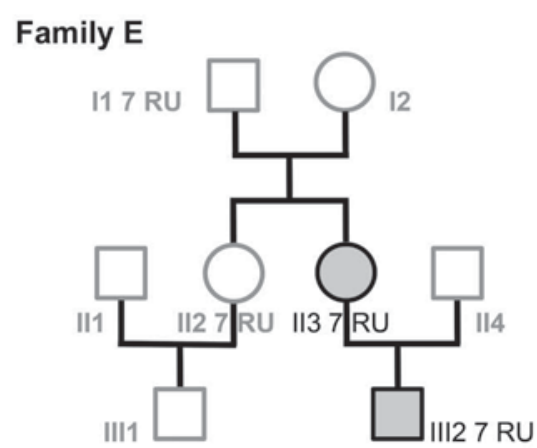

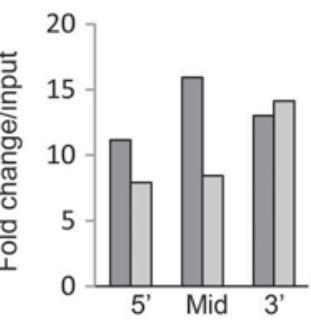

$\square$ II3 $\square$ III2

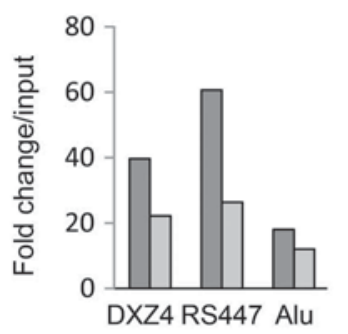

Bisulfite
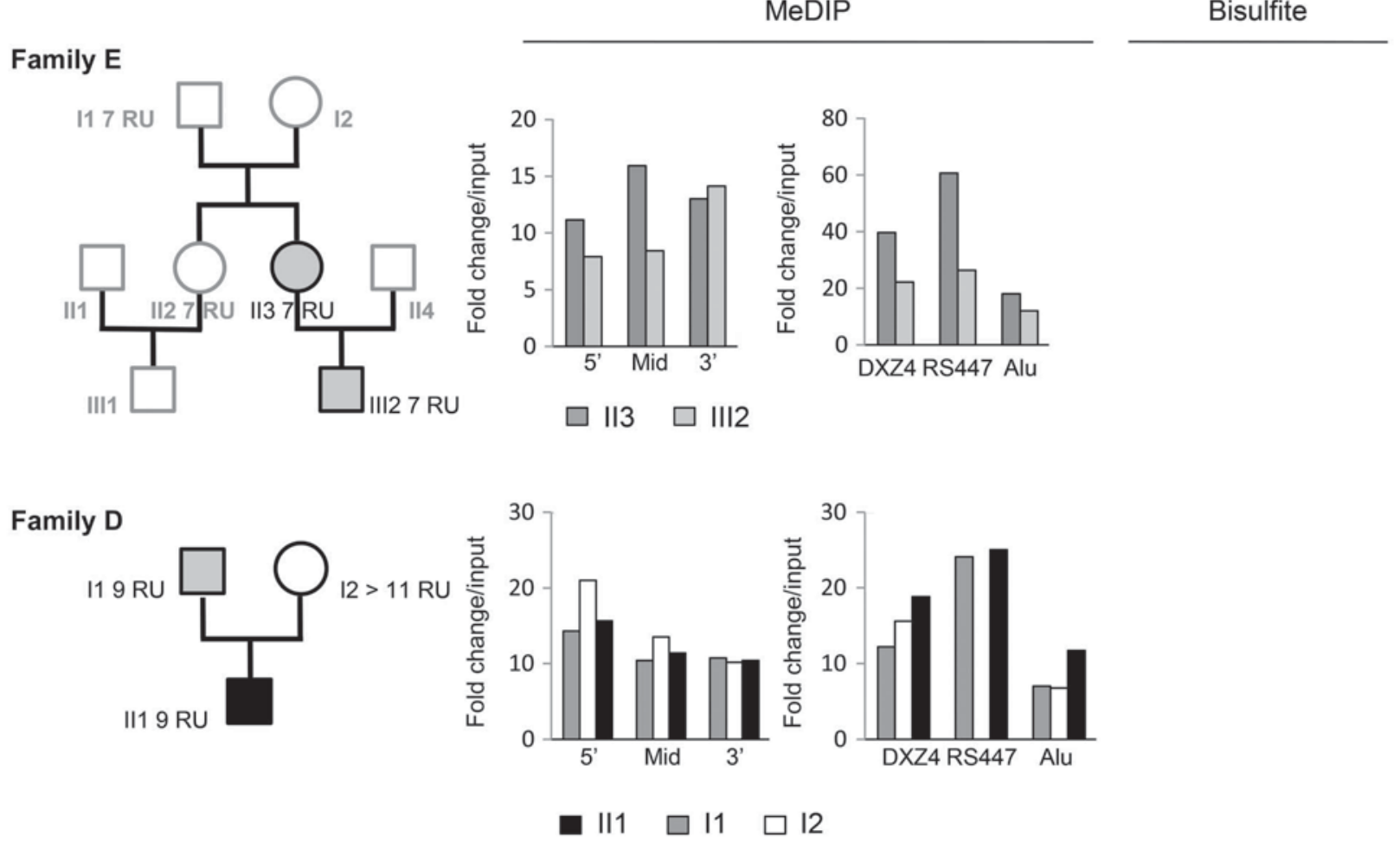

Family B
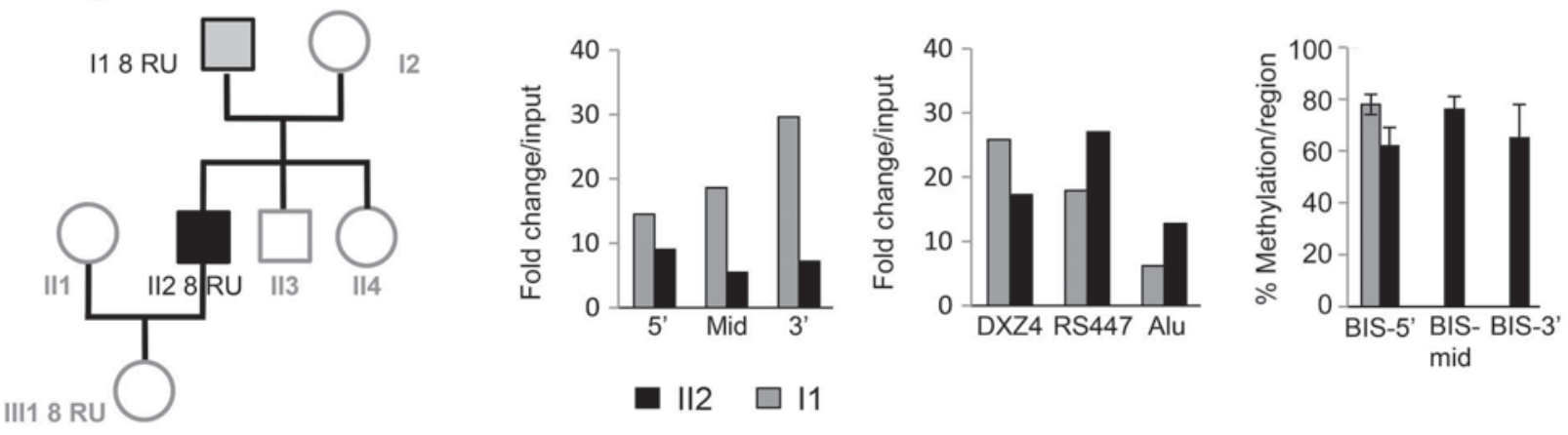

III1 8 RU

Family C
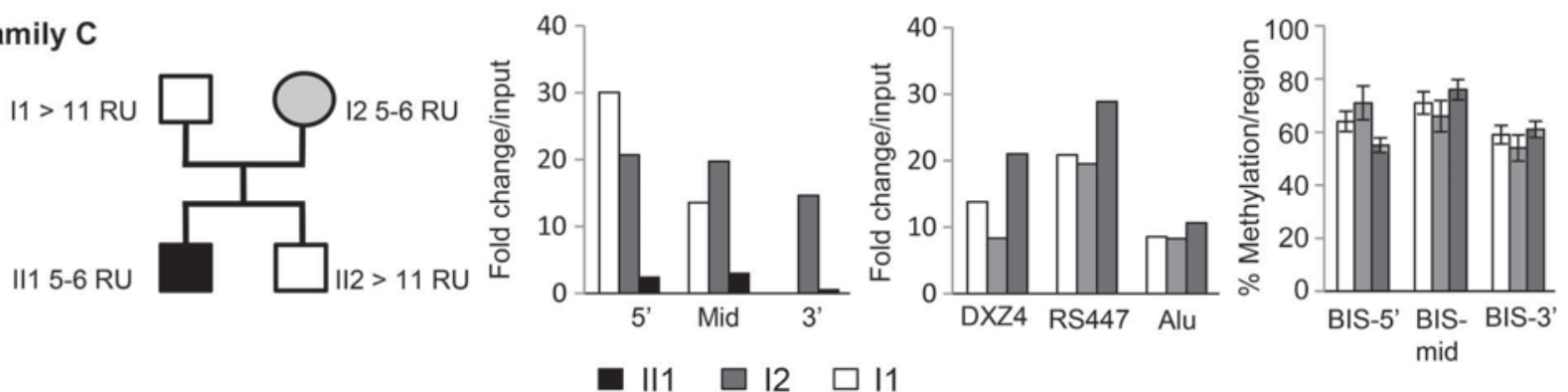

We compared the level of methylation of D4Z4 (left graphs) and other repetitive sequences (middle graph) for 4 families for which variable penetrance has been reported from one generation to another between nonsymptomatic carrier parents (in whom mosaicism has been excluded above a $4 \%$ level) and affected descendants (E, D, B, and C) by methylated DNA immunoprecipitation (MeDIP) or by bisulfite for 2 families (B and C). For MeDIP, histograms display the enrichment in methylated DNA/CpG normalized to chromosome 5 (y-axis). For bisulfite (right panel), histograms represent the mean percentage of methylation/individual (y-axis) in the different subgroups of samples for the BIS-5' region (left), BIS-mid (middle), and BIS-3' (right) with errors bars representing the SD. The values obtained for the different individuals are included in the data presented in figures 3 and 5. 
Figure 5 Comparison of patients with FSHD1 and asymptomatic carriers reveals differences in the methylation of the proximal region of $D 4 Z 4$

A
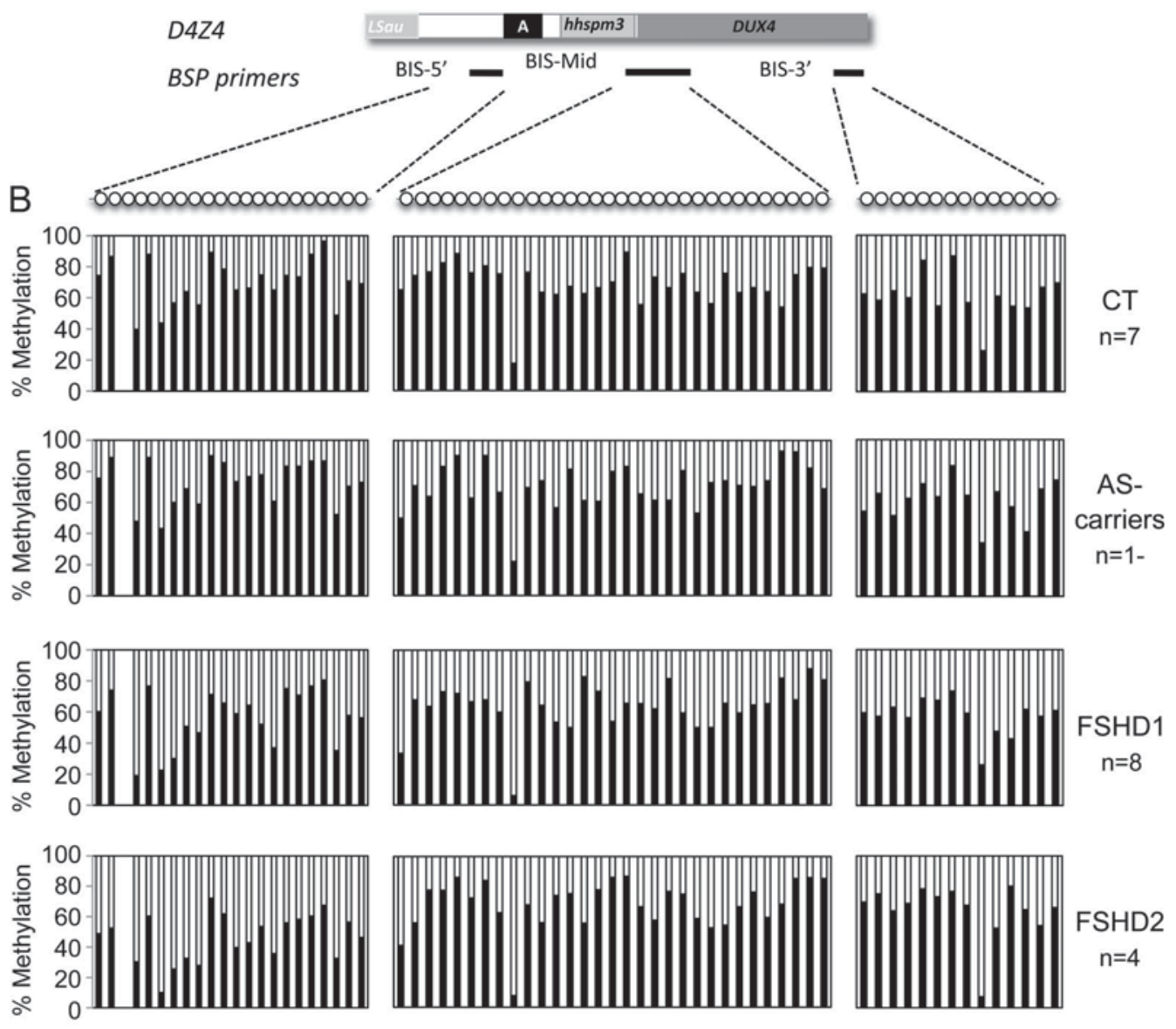

C
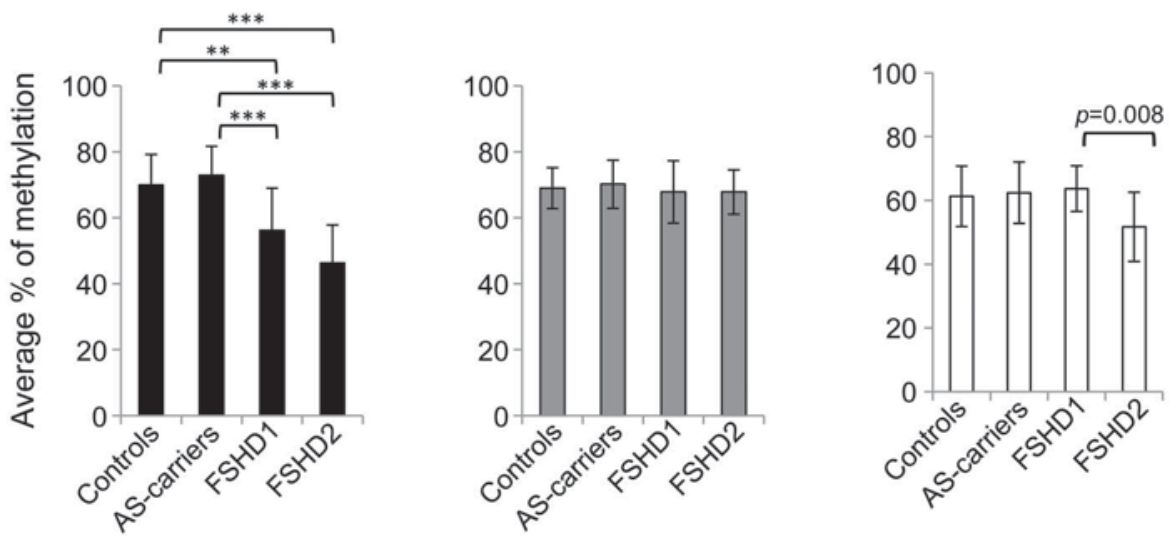

(A) The absolute level of DNA methylation at a single nucleotide level was determined after sodium bisulfite sequencing for 3 different regions within the D4Z4 sequence: BIS-5' (21 CpGs, represented by dots), BIS-mid (31 CpGs), and BIS-3' (14 CpGs). (B) For each sequence, 7 controls (CT), 10 asymptomatic (AS) carriers, 8 patients with facioscapulohumeral dystrophy (FSHD)1, and 4 patients with FSHD2 were analyzed (detailed results are presented in figures e-4-e-7). Histogram bars represent the percentage of methylated (black) or unmethylated (white) CpG for each position in the 3 regions. (C) Histograms display the mean percentage of methylation/individual (y-axis) in the different subgroups of samples for the BIS- $5^{\prime}$ region (left), BIS-mid (middle), and BIS-3' (right) with errors bars representing the SD among the different DNA molecules analyzed. Datasets were compared with the Kruskal-Wallis nonparametric test, and brackets identify the groups in which values are significantly different based on post hoc Dunn comparison and Bonferroni correction $(* * p=0.001 ; * * * p<0.001$ for the 5' sequence). 
DNA methylation in FSHD2 in the proximal part of the repeat (figure 5C). Moreover, combination of the percentage of methylation for the different clones in the different subgroups revealed that the level of methylation is similar in FSHD1 and FSHD2. Significant differences were observed between controls and FSHD1 or FSHD2 for the most proximal sequence (BIS-5') (figure 5, B and C). Strikingly, using a nonparametric Kruskal-Wallis test, a significant difference in the percentage of methylated DNA molecules was also observed between asymptomatic FSHD carriers and patients with FSHD1 or FSHD2 (figure 5C). In addition to the proximal hypomethylated region, a significant hypomethylation was also observed in the $3^{\prime}$ end of the D $4 Z 4$ repeat in FSHD2 compared with FSHD1 (figure 5C). PCA between the level of methylation in the different $D 4 Z 4$ subregions with sex, age, number of repeats, and presence or absence of symptoms confirmed that the decreased methylation level in the $5^{\prime}$ region negatively correlated with the presence of symptoms by comparing the 4 different subgroups (Pearson correlation coefficient, $r=-0.593$; $p=0.02$ ) or by comparing patients with FSHD1, asymptomatic carriers, and controls (Pearson correlation coefficient, $r=-0.514$; $p=0.02$ ). We did not observe any strong correlation among the level of methylation, number of $D 4 Z 4$ repeats, age, or sex (table e-8).

Moreover, PCA taking into account the presence or absence of symptoms and the percentage of methylation for each individual $\mathrm{CpG}$ revealed that the disease is mainly associated with a decrease methylation of a few CpGs within the $5^{\prime}$ region $(\mathrm{CpG}, 1 ; 2 ; 5 ; 7 ; 10$; 11; 15; 20; Pearson correlation coefficient $-0.379<$ $r<-0.498 ; 0.047<p$ value $<0.007$ )

Our results indicate that the degree of $D 4 Z 4$ hypomethylation is not directly correlated with shortening of the array but suggest a direct correlation between hypomethylation and the presence of clinical signs of the disease. Furthermore, hypomethylation is not uniformly spread across the $D 4 Z 4$ repeat but confined to discrete regions or specific sites, suggesting a complex regulatory mechanism, which remains to be investigated.

DISCUSSION We aimed to identify regions within D4Z4 differentially methylated in individuals with FSHD and asymptomatic individuals carrying a shortened pathogenic array. By investigating a large collection of 95 samples including controls, individuals with FSHD, asymptomatic carriers, and patients with FSHD2 using 2 methods, immunoprecipitation of methylated CpGs (MeDIP) and sodium bisulfite sequencing, we reasoned that specific methylation changes might shed light on disease-associated alterations linked to disease penetrance or onset.
So far, methylation status of either the most proximal or the most distal $D 4 Z 4$ repeat has been mainly analyzed by Southern blotting after digestion with 3 methylation-sensitive enzymes, BsaAI, FseI, and CpoI, allowing analysis of only a few CpG sites. In patients with FSHD, hybridization with a DNA probe corresponding to the proximal unique sequence upstream of the first $D 4 Z 4$ repeat ${ }^{9-11,13,23}$ shows a decreased methylation of approximately $10 \%$ to $30 \%$ compared with controls, indicating that epigenetic changes are involved in the pathology.

Both sodium bisulfite sequencing and MeDIP remain more resolutive than approaches based on the capacity of methyl-sensitive restriction enzymes to cleave a single recognition site within a specific DNA sequence. Because of the high CpG density of the $D 4 Z 4$ repeat, MeDIP is well adapted, while this method is less sensitive for bulk DNA in which CpG sites are more dispersed. ${ }^{24,25}$ By MeDIP, we observed significant differences between controls and carriers of a short $4 \mathrm{qA}$ allele ( $<11$ units) but asymptomatic for the pathology and individuals with FSHD. However, this method did not permit identification of specific hypomethylated sites.

Therefore, we also assessed the methylation level across the $D 4 Z 4 / \mathrm{CpG} / \mathrm{DNA}$ molecule at a single base resolution by bisulfite sequencing. This assay revealed a marked hypomethylation in the proximal and distal part of D $4 Z 4$ for FSHD2 and the proximal part of the repeat for FSHD1 with specific differences between asymptomatic carriers and patients with FSHD even in the same family. These differences seem specific for $D 4 Z 4$ because they were not observed genome-wide for dispersed $A l u \mathrm{I}$ elements or for other clustered tandem macrosatellite repeats (RS447, DXZ4).

In the majority of individuals carrying 1 to $3 D 4 Z 4$ units, penetrance is considered as almost complete at age 50 years while in patients carrying between 4 and 8 repeated units, the risk of developing symptoms is $55 \%$ to $63 \%$ at the age of $50 .{ }^{26}$ Differential methylation is also observed between symptomatic and asymptomatic individuals with a low number of $D 4 Z 4$ units (5 units) and in individuals for whom first-degree relatives have been diagnosed at a younger age. The differences in the degree of hypomethylation are not biased by the age at onset as described in the literature for heterochromatic regions and repetitive sequences because, on average, individuals in the asymptomatic group are older (mean age $=52.6$ years) than in the control (mean age $=47.5$ years) or FSHD1 (mean age $=43.6$ years) groups.

FSHD is characterized by a highly variable disease progression, incomplete penetrance, and a marked interindividual and intrafamilial heterogeneity. ${ }^{15,16,27,28}$ By analyzing the degree of methylation in different families, we also show that methylation level is not 
stably transmitted together with the number of repeats among individuals in the same family but that hypomethylation is mainly observed in individuals harboring FSHD symptoms. However, our results also suggest that the differential methylation between asymptomatic carriers and patients with FSHD1 might be influenced by sex, but this aspect would require further investigations on a larger cohort.

A key question regarding FSHD is whether hypomethylation is a cause or consequence of $D 4 Z 4$ array shortening in FSHD1. Here, hypomethylation is not directly correlated with the number of repeats suggesting that in FSHD1, hypomethylation is not a mere consequence of $D 4 Z 4$ array shortening and that the clinical phenotype might be determined by the epigenetic changes rather than Mendelian inheritance in both FSHD1 nonsymptomatic carriers of short D $4 Z 4$ stretches and patients with FSHD2 who have normal $D 4 Z 4$ arrays.

The focal rather than global and random hypomethylation of D4Z4 in patients with FSHD1 and FSHD2 $^{22}$ in the proximal or distal part of the repeat suggests an active and targeted mechanism. The current hypothesis explaining the FSHD pathomechanism is the production in affected individuals of a long DUX4 transcript encoded by the last D4Z4 and the distal 4qA region. We did not observe any significant hypomethylation in the region encompassing the DUX4 promoter sequence (mid) and the DBE element, ${ }^{29,30}$ but it remains to be determined whether focal demethylation of only a few CpGs upstream or downstream induces DUX4 expression in patients with FSHD or whether hypomethylation across the whole D4Z4 array globally modifies the chromatin of the $4 \mathrm{q} 35$ region and expression of other genes. Notably, we also observed a high rate of sequence variation at CpGs especially in the mid (18\%) and 3' (16\%) regions, suggesting a high level of polymorphism throughout the $D 4 Z 4$ sequences, which might also affect the production of the various $D 4 Z 4$ transcripts.

Overall, the presence of discrete sites hypomethylated in patients but not in controls or asymptomatic carriers opens new perspectives for mechanistic investigations and identification of regulatory proteins or processes implicated in the pathogenesis and future development of animal models of the disease.

Furthermore, even if DNA methylation is a heritable epigenetic mark, it is also reversible throughout life especially in response to lifestyle, diet, and environment, ${ }^{31}$ but can reflect the susceptibility to diseases as exemplified by a number of studies on monozygotic twins, a phenomenon referred to as "epigenetic drift." 32

Our results indicate that $D 4 Z 4$ methylation is not stably transmitted through generations and that incomplete penetrance in asymptomatic carriers is associated with a level of $D 4 Z 4$ methylation similar to controls suggesting variability in the transmission of this epigenetic mark and/or somatic variability in affected individuals. At the clinical level, the next step would be to design more targeted approaches that could be applied systematically to patient samples in routine diagnosis, especially in the case of patients with FSHD2 for which the molecular diagnosis is often complex to interpret in the absence of $D 4 Z 4$ array shortening.

In the context of FSHD, understanding the dynamics of the hypomethylation process may thus provide us with new insights on the disease pathomechanism, and our study also suggests that, in combination with the different $4 \mathrm{q} 35$ haplotypes or single nucleotide polymorphisms, ${ }^{26,33-35}$ the degree of methylation might reflect part of the heterogeneity in the course of the disease and act as a modifier in the clinical expression of this complex muscular dystrophy.

\section{AUTHOR CONTRIBUTIONS}

Marie-Cécile Gaillard: set up the experimental conditions, performed the experiments, and analyzed and organized the data. Stéphane Roche: set up the conditions and analyzed the data. Camille Dion, Armand Tasmadjian, and Gwenaëlle Bouget: performed the experiments and analyzed the data. Emmanuelle Salort-Campana: provided patient samples and analyzed the data. Catherine Vovan, Charlene Chaix, and Natacha Broucqsault: processed patient samples and analyzed the results. Julia Morere: provided and processed patient samples. Francesca Puppo: provided and processed patient samples and analyzed the data. Marc Bartoli, Nicolas Levy, and Rafaëlle Bernard, and Shahram Attarian: provided patient samples and analyzed the data. Karine Nguyen: contributed to the design and coordination of the project and analysis of the data, provided patient samples. Frédérique Magdinier: designed and coordinated the project, performed the statistical analysis, analyzed the data, and wrote the manuscript.

\section{ACKNOWLEDGMENT}

The authors are indebted to all families for participating in this study.

\section{STUDY FUNDING}

Funded by FSHD Global (Australia), the FSH Society (United States), Association Française contre les Myopathies (AFM), and an ANR grant (FSHDecrypt, ANR-09-GENO-038) (to F.M.). N.B. and F.P. are recipients of a fellowship from AFM (Association Française contre les Myopathies). S.R. is the recipient of a fellowship from ANR (FSHDecrypt, ANR-09-GENO-038). M.-C.G. is the recipient of a fellowship from the FSH Society.

\section{DISCLOSURE}

The authors report no disclosures relevant to the manuscript. Go to Neurology.org for full disclosures.

Received November 20, 2013. Accepted in final form April 29, 2014.

\section{REFERENCES}

1. Smith ZD, Meissner A. DNA methylation: roles in mammalian development. Nat Rev Genet 2013;14:204-220.

2. Cantone I, Fisher AG. Epigenetic programming and reprogramming during development. Nat Struct Mol Biol 2013;20:282-289.

3. Toyota M, Ohe-Toyota M, Ahuja N, Issa JP. Distinct genetic profiles in colorectal tumors with or without the 
CpG island methylator phenotype. Proc Natl Acad Sci USA 2000;97:710-715.

4. Bocker MT, Hellwig I, Breiling A, Eckstein V, Ho AD, Lyko F. Genome-wide promoter DNA methylation dynamics of human hematopoietic progenitor cells during differentiation and aging. Blood 2011;117:e182-e189.

5. Wilson AS, Power BE, Molloy PL. DNA hypomethylation and human diseases. Biochim Biophys Acta 2007;1775: 138-162.

6. Bergman Y, Cedar H. DNA methylation dynamics in health and disease. Nat Struct Mol Biol 2013;20: 274-281.

7. Lander ES, Linton LM, Birren B, et al. Initial sequencing and analysis of the human genome. Nature 2001;409: 860-921.

8. Kondo T, Bobek MP, Kuick R, et al. Whole-genome methylation scan in ICF syndrome: hypomethylation of non-satellite DNA repeats D4Z4 and NBL2. Hum Mol Genet 2000;9:597-604.

9. van Overveld PG, Lemmers RJ, Sandkuijl LA, et al. Hypomethylation of D4Z4 in 4q-linked and non-4q-linked facioscapulohumeral muscular dystrophy. Nat Genet 2003;35:315-317.

10. Sacconi S, Camano P, de Greef JC, et al. Patients with a phenotype consistent with facioscapulohumeral muscular dystrophy display genetic and epigenetic heterogeneity. J Med Genet 2012;49:41-46.

11. van Overveld PG, Enthoven L, Ricci E, et al. Variable hypomethylation of D4Z4 in facioscapulohumeral muscular dystrophy. Ann Neurol 2005;58:569-576.

12. de Greef JC, Lemmers RJ, Camano P, et al. Clinical features of facioscapulohumeral muscular dystrophy 2. Neurology 2010;75:1548-1554.

13. de Greef JC, Lemmers RJ, van Engelen BG, et al. Common epigenetic changes of D4Z4 in contractiondependent and contraction-independent FSHD. Hum Mutat 2009;30:1449-1459.

14. Lemmers RJ, Tawil R, Petek LM, et al. Digenic inheritance of an SMCHD1 mutation and an FSHD-permissive D4Z4 allele causes facioscapulohumeral muscular dystrophy type 2. Nat Genet 2012;44:1370-1374.

15. Tonini MM, Passos-Bueno MR, Cerqueira A, Matioli SR, Pavanello R, Zatz M. Asymptomatic carriers and gender differences in facioscapulohumeral muscular dystrophy (FSHD). Neuromuscul Disord 2004;14:33-38.

16. Lunt PW, Jardine PE, Koch MC, et al. Correlation between fragment size at D4F104S1 and age at onset or at wheelchair use, with a possible generational effect, accounts for much phenotypic variation in 4q35-facioscapulohumeral muscular dystrophy (FSHD). Hum Mol Genet 1995;4:951-958.

17. Goto Y, Feil R. Genomic imprinting and its effects on genes and chromosomes in mammals. Methods Mol Biol 2004;240:53-75.

18. Sakellariou P, Kekou K, Fryssira H, et al. Mutation spectrum and phenotypic manifestation in FSHD Greek patients. Neuromuscul Disord 2012;22:339-349.

19. Tawil R, Forrester J, Griggs RC, et al. Evidence for anticipation and association of deletion size with severity in facioscapulohumeral muscular dystrophy. The FSH-DY Group. Ann Neurol 1996;39:744-748.

20. Frommer M, McDonald LE, Millar DS, et al. A genomic sequencing protocol that yields a positive display of 5 methylcytosine residues in individual DNA strands. Proc Natl Acad Sci USA 1992;89:1827-1831.

21. Rhee I, Bachman KE, Park BH, et al. DNMT1 and DNMT3b cooperate to silence genes in human cancer cells. Nature 2002;416:552-556.

22. Hartweck LM, Anderson LJ, Lemmers RJ, et al. A focal domain of extreme demethylation within D4Z4 in FSHD2. Neurology 2013;80:392-399.

23. de Greef JC, Wohlgemuth M, Chan OA, et al. Hypomethylation is restricted to the $\mathrm{D} 4 \mathrm{Z} 4$ repeat array in phenotypic FSHD. Neurology 2007;69:1018-1026.

24. Cross SH, Charlton JA, Nan X, Bird AP. Purification of CpG islands using a methylated DNA binding column. Nat Genet 1994;6:236-244.

25. Horard B, Eymery A, Fourel G, et al. Global analysis of DNA methylation and transcription of human repetitive sequences. Epigenetics 2009;4:339-350.

26. Ricci G, Scionti I, Sera F, et al. Large scale genotypephenotype analyses indicate that novel prognostic tools are required for families with facioscapulohumeral muscular dystrophy. Brain 2013;136:3408-3417.

27. Zatz M, Marie SK, Passos-Bueno MR, et al. High proportion of new mutations and possible anticipation in Brazilian facioscapulohumeral muscular dystrophy families. Am J Hum Genet 1995;56:99-105.

28. Pastorello E, Cao M, Trevisan CP. Atypical onset in a series of 122 cases with FacioScapuloHumeral Muscular Dystrophy. Clin Neurol Neurosurg 2012;114:230-234.

29. Cabianca DS, Casa V, Bodega B, et al. A long ncRNA links copy number variation to a polycomb/trithorax epigenetic switch in FSHD muscular dystrophy. Cell 2012; 149:819-831.

30. Gabellini D, Green MR, Tupler R. Inappropriate gene activation in FSHD: a repressor complex binds a chromosomal repeat deleted in dystrophic muscle. Cell 2002;110: 339-348.

31. Doi A, Park IH, Wen B, et al. Differential methylation of tissue- and cancer-specific CpG island shores distinguishes human induced pluripotent stem cells, embryonic stem cells and fibroblasts. Nat Genet 2009;41:1350-1353.

32. Fraga MF, Ballestar E, Paz MF, et al. Epigenetic differences arise during the lifetime of monozygotic twins. Proc Natl Acad Sci USA 2005;102:10604-10609.

33. Scionti I, Greco F, Ricci G, et al. Large-scale population analysis challenges the current criteria for the molecula diagnosis of fascioscapulohumeral muscular dystrophy. Am J Hum Genet 2012;90:628-635.

34. Caruso N, Herberth B, Bartoli M, et al. Deregulation of the protocadherin gene FAT1 alters muscle shapes: implications for the pathogenesis of facioscapulohumeral dystrophy. PLoS Genet 2013;9:e1003550.

35. Sacconi S, Lemmers RJ, Balog J, et al. The FSHD2 gene SMCHD1 is a modifier of disease severity in families affected by FSHD1. Am J Hum Genet 2013;93:744-751. 